\title{
Acanthamoeba hatchetti
}

National Cancer Institute

\section{Source}

National Cancer Institute. Acanthamoeba hatchetti. NCI Thesaurus. Code C119301.

A species of motile, unicellular eukaryotic amoebae within the family of

Acanthamoebidae. A. hatchetti is placed in morphological group II with a 18s rRNA gene sequence type of T11. Members of this group are characterized by the presence of wrinkled ectocysts and endocysts which could be stellate, polygonal, triangular, or oval. This species is a human pathogen and may cause granulomatous amoebic encephalitis and amoebic keratitis. It may also cause amoebic placentitis in horses. 